OPEN ACCESS

Edited by:

Jose Eduardo Villarreal Barajas,

Royal Devon and Exeter Hospital,

United Kingdom

Reviewed by:

Kevin Martell,

University of Calgary, Canada

Zhang Yi,

Sichuan University, China

Leyla Moghaddasi,

University of Melbourne, Australia

*Correspondence:

Fuquan Zhang

zhangfuquan3@126.com

Jie Qiu

qiujie@pumch.cn

${ }^{\dagger}$ These authors share first authorship

Specialty section:

This article was submitted to

Radiation Oncology,

a section of the journal

Frontiers in Oncology

Received: 29 April 2021

Accepted: 29 July 2021

Published: 19 August 2021

Citation:

Liu Z, Chen W, Guan H, Zhen H,

Shen J, Liu X, Liu A, Li R, Geng J, You J, Wang W, LiZ, Zhang Y, Chen Y,

Du J, Chen Q, Chen Y, Wang S,

Zhang F and Qiu J (2021) An

Adversarial Deep-Learning-Based

Model for Cervical Cancer CTV

Segmentation With Multicenter Blinded Randomized

Controlled Validation.

Front. Oncol. 11:702270.

doi: 10.3389/fonc. 2021.702270

\section{An Adversarial Deep-Learning-Based Model for Cervical Cancer CTV Segmentation With Multicenter Blinded Randomized Controlled Validation}

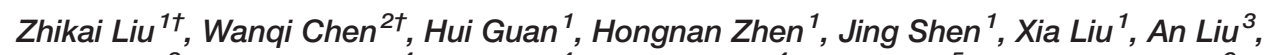
Richard $L^{3}{ }^{3}$, Jianhao Geng ${ }^{4}$, Jing You ${ }^{4}$, Weihu Wang ${ }^{4}$, Zhouyu $L^{5}{ }^{5}$, Yongfeng Zhang ${ }^{6}$, Yuanyuan Chen ${ }^{7}$, Junjie $\mathrm{Du}^{8}$, Qi Chen ${ }^{9}$, Yu Chen ${ }^{9}$, Shaobin Wang ${ }^{9}$, Fuquan Zhang ${ }^{1 *}$ and Jie Qiu ${ }^{1 *}$

\begin{abstract}
${ }^{1}$ Department of Radiation Oncology, Peking Union Medical College Hospital, Chinese Academy of Medical Sciences and Peking Union Medical College, Beijing, China, ${ }^{2}$ Department of Nuclear Medicine, Sun Yat-Sen University Cancer Center, Guangzhou, China, ${ }^{3}$ Department of Radiation Oncology, City of Hope National Medical Center, Duarte, CA, United States, ${ }^{4}$ Key Laboratory of Carcinogenesis and Translational Research (Ministry of Education/Beijing), Department of Radiation Oncology, Peking University Cancer Hospital and Institute, Beijing, China, ${ }^{5}$ Department of Radiation Oncology, Affiliated Cancer Hospital \& Institute of Guangzhou Medical University, Guangzhou, China, ${ }^{6}$ Department of Radiation Oncology, The Fourth Hospital of Jilin University (FAW General Hospital), Jilin, China, ${ }^{7}$ Oncology Department, Cangzhou Hospital of Integrated Traditional Chinese and Western Medicine, Hebei, China, ${ }^{8}$ Department of Radiation Oncology, Yangquan First People's Hospital, Shanxi, China, ${ }^{9}$ Research and Development Department, MedMind Technology Co., Ltd., Beijing, China
\end{abstract}

Purpose: To propose a novel deep-learning-based auto-segmentation model for CTV delineation in cervical cancer and to evaluate whether it can perform comparably well to manual delineation by a three-stage multicenter evaluation framework.

Methods: An adversarial deep-learning-based auto-segmentation model was trained and configured for cervical cancer CTV contouring using CT data from 237 patients. Then CT scans of additional 20 consecutive patients with locally advanced cervical cancer were collected to perform a three-stage multicenter randomized controlled evaluation involving nine oncologists from six medical centers. This evaluation system is a combination of objective performance metrics, radiation oncologist assessment, and finally the head-tohead Turing imitation test. Accuracy and effectiveness were evaluated step by step. The intra-observer consistency of each oncologist was also tested.

Results: In stage-1 evaluation, the mean DSC and the 95HD value of the proposed model were 0.88 and $3.46 \mathrm{~mm}$, respectively. In stage-2, the oncologist grading evaluation showed the majority of Al contours were comparable to the GT contours. The average CTV scores for Al and GT were 2.68 vs. 2.71 in week $0(P=.206)$, and 2.62 vs. 2.63 in week $2(P=.552)$, with no significant statistical differences. In stage-3, the Turing imitation test showed that the percentage of Al contours, which were judged to be better than GT contours by $\geq 5$ oncologists, was $60.0 \%$ in week 0 and $42.5 \%$ in week 2 . Most oncologists demonstrated good consistency between the 2 weeks $(P>0.05)$. 


\section{Conclusions: The tested Al model was demonstrated to be accurate and comparable to} the manual CTV segmentation in cervical cancer patients when assessed by our threestage evaluation framework.

Keywords: deep-learning, auto-segmentation, evaluation, cervical cancer, radiotherapy, clinical target volume

\section{INTRODUCTION}

Cervical cancer (CC) remains one of the leading causes of cancer-related deaths in women worldwide (1). The majority of cervical cancer cases are diagnosed at the locally advanced stage in developing countries (2). External beam radiotherapy (EBRT) with concurrent chemotherapy followed by brachytherapy, also known as radical radiotherapy (RT), is the standard treatment for locally advanced cervical cancer (3) and has been shown to be effective in decreasing the risk of pelvic and vaginal vault recurrence (4).

Accurate and individualized clinical target volume (CTV) definition is vitally important for the definitive treatment of CC (5). During the past few years, a few high-performance deeplearning models based on convolutional neural networks (CNNs) have made tremendous progress and shown promise to serve as excellent assistance for target segmentation (6-12).

A recent study has first applied a deep-learning-based method called DpnUNet to CTV segmentation in cervical cancer. The authors' previous experimental results demonstrated that $88.65 \%$ of the contours generated by DpnUNet were acceptable for clinical usage (13). The mean dice similarity coefficient (DSC) and the $95^{\text {th }}$ Hausdorff distance (95HD) were 0.86 and 5.34 for the delineated CTVs. However, there are still some glaring deficits. First, performance metrics such as mean DSC and 95HD are objective and offer good reproducibility (14-17), but do not incorporate physician's judgment and may not effectively evaluate for accuracy and applicability in a practical clinical context. Second, although the subjective oncologists' assessments showed that most predicted contours were acceptable for clinical usage when a head-to-head comparison was conducted between manual and AI-generated contours in the same CT slice, the DpnUNet model performed inferiorly. Therefore, it indicated that the currently proposed models did not perform exactly comparably well to manual delineations in clinical practice. Moreover, it seems that the current evaluation system for automatic segmentation models remains limited and insufficient.

Given the aforementioned reasons, a novel adversarial deeplearning-based auto-segmentation model is hence proposed for CTV delineation in cervical cancer. Then a challenging three-stage multicenter randomized controlled evaluation system is designed to directly validate the model and to minimize the inter- and intra-

Abbreviations: CC, cervical cancer; RT, radiotherapy; EBRT, external beam radiotherapy; CTV, clinical target volume; ROIs, regions of interest; CNNs, convolutional neural networks; DPN, dual path network; GT, ground truth contouring; AI, artificial Intelligence; DICOM, digital imaging and communications in medicine; ESTRO, European Society for Radiotherapy and Oncology; RTOG, Radiation Therapy Oncology Group; DSC, Dice similarity coefficient; $95 \mathrm{HD}$, the $95^{\text {th }}$ Hausdorff distance; IMRT, intensity modulated radiation therapy. observer variability. This evaluation system is a combination of objective performance metrics, subjective radiation oncologist assessment, and finally, the Turing imitation test. Accuracy and effectiveness were evaluated step by step.

\section{MATERIALS AND METHODS}

\section{Network Architecture}

CTVs are challenged to be evaluated with mathematical indicators due to fuzzy boundaries and large variations among different centers and observers. Inspired by a previously described work (18), an adversarial training approach based on the typical segmentation model is proposed to achieve similar performance between CTVs delineated by the proposed model and the oncologists. The overall architecture is shown in Figure $\mathbf{1 .}$

The proposed model is based on DpnUNet (13), which originated from the architecture of U-Net (19), but replaces all the encoder and decoder components with DPN components. Considering that the original DpnUNet is still underperforming compared with manual delineation in clinical practice, an extra convolutional layer is added at the end of DpnUNet, in which the output channels are one and the kernel size is $1 \times 1$. A ResNet-10 with binary classification is used as the discriminator network (20). Since the discriminator is trained to identify the input segmentation generated by the model or delineated by oncologists, it will feedback the results to the model to promote similarities between the predicted CTVs and manual delineations.

The model was trained and tested using sets of CT data from 237 patients with locally advanced cervical cancer in our center with a GTX 1080GPU. All data using oral and IV contrast were constructed with a size of $512 \times 512$ pixels and acquired with a Brilliance CT Big Bore (Philips Healthcare, Best, Netherlands). The proposed model was trained over 50 circles to select the best model according to the lowest validation loss score.

\section{Data Acquisition}

To perform the three-stage evaluation, CT scans of a separate set of 20 new validation patients with locally advanced cervical cancer undergoing intensity-modulated radiation therapy (IMRT) were collected from November 2018 to December 2018 at the Peking Union Medical College Hospital. All patients were diagnosed with FIGO stage IB1-IIIC1 and/or node metastasis positive $(\mathrm{N}+) \mathrm{CC}$, treated with EBRT and radical $\mathrm{RT}$. The average age \pm standard deviation of these patients was $51.90 \pm 12.63$ years old.

CTV contours of 20 patients were redefined and re-delineated manually by radiation oncologists following the updated Radiation Therapy Oncology Group (RTOG) protocols 


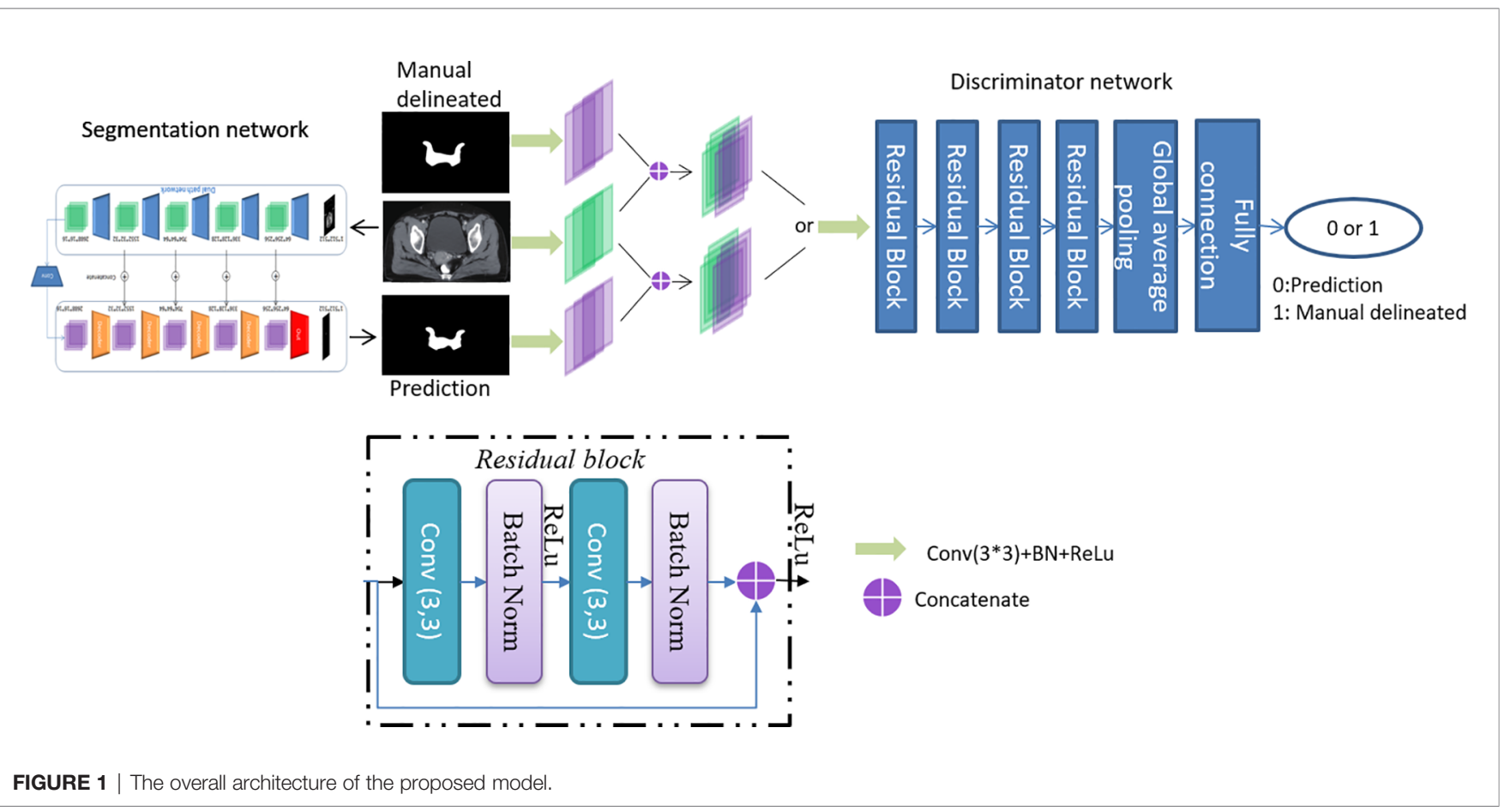

(21-23). The CTV contours included the whole cervix, uterus, parametrium, vagina for $2 \mathrm{~cm}$ below GTV, and the elective nodal volume. All the contours were first reviewed by two senior radiation oncologists with more than 10 years of experience in radiotherapy specialized in cervical cancer at the Peking Union Medical College Hospital. To ensure the delineation quality of the human-generated CTV, the delineated contours were reviewed, modified, and approved collaboratively by a radiation oncologist committee consisting of eight senior oncologists at the Peking Union Medical College Hospital. The dataset of CT scans of 20 patients was used as a testing set of the proposed model to obtain artificial intelligence-generated contouring (AI) for performance assessment, of which 10 patients were randomly selected by Fisher-Yates shuffle for oncologist evaluation and the other 10 patients for the Turinglike test.

\section{The Three-Stage Multicenter Randomized Controlled Evaluation}

\section{Stage 1: Performance Metrics}

The flowchart of the three-level multicenter randomized controlled evaluation is shown in Figure 2. During the firststage test, the Dice similarity coefficient (DSC) and the 95th percentile Hausdorff distance (95HD) were used to quantify the performance of the proposed model objectively.

The DSC was used to measure the spatial overlap between AI and GT contours, which is defined in Eq. (1).

$$
\operatorname{DSC}(\mathrm{A}, \mathrm{B})=\frac{2|\mathrm{~A} \cap \mathrm{B}|}{|\mathrm{A}|+|\mathrm{B}|}
$$

Where $A$ represents the volume of human-generated contour; $B$ is the volume of an $\mathrm{AI}$ contour; and $\mathrm{A} \cap \mathrm{B}$ is the intersection volume that $\mathrm{A}$ and $\mathrm{B}$ have in common. The DSC value is between 0 and $1(0=$ no overlap, $1=$ complete overlap $)$.

The 95HD is defined as follows:

$$
95 \mathrm{HD}(\mathrm{A}, \mathrm{B})=\max \left(\mathrm{h}(\mathrm{A}, \mathrm{B}), \mathrm{h}(\mathrm{B}, \mathrm{A}), 95^{\mathrm{th}}\right)
$$

$$
\begin{gathered}
\mathrm{HD}(\mathrm{A}, \mathrm{B})=\max (\mathrm{h}(\mathrm{A}, \mathrm{B}), \mathrm{h}(\mathrm{B}, \mathrm{A}))=\max (\operatorname{maxmin}|| \mathrm{a}-\mathrm{b} \|, \operatorname{maxmin} \mid \\
|\mathrm{b}-\mathrm{a}| \mid) \mathrm{a} \in \mathrm{Ab} \in \mathrm{B} \quad \mathrm{b} \in \mathrm{Ba} \in \mathrm{A}
\end{gathered}
$$

$\|\bullet\|$ means the Euclidean norm of the points of $A$ and $B$. The HD in $\mathrm{mm}$ depicts the maximum mismatch between $\mathrm{A}$ and $\mathrm{B}$. When the $\mathrm{HD}$ value decreases, the overlap between $\mathrm{A}$ and $\mathrm{B}$ increases. The mean and standard deviation were calculated.

\section{Stage 2: Oncologist Evaluation}

Ten cases from the testing set were randomly collected for oncologist evaluation. Twenty slices from each case were randomly extracted by Fisher-Yates shuffle, of which 10 slices were randomly selected to show GT contours, and the others were overlaid with AI contours. In total, 200 slices were obtained (AI: $10 \times 10=100$ slices vs. GT: $10 \times 10=100$ slices) and then randomly assigned to nine experienced radiation oncologists from six different cancer centers with more than 10 years of clinical experience in cervical cancer. The dataset of 200 randomized slices was evaluated by each oncologist slice by slice. The contours were graded in four scores: 3 points (No revision), 2 points (Minor revision), 1 point (Major revision), and 0 points (Rejection). The rubric is shown in Table 1. 


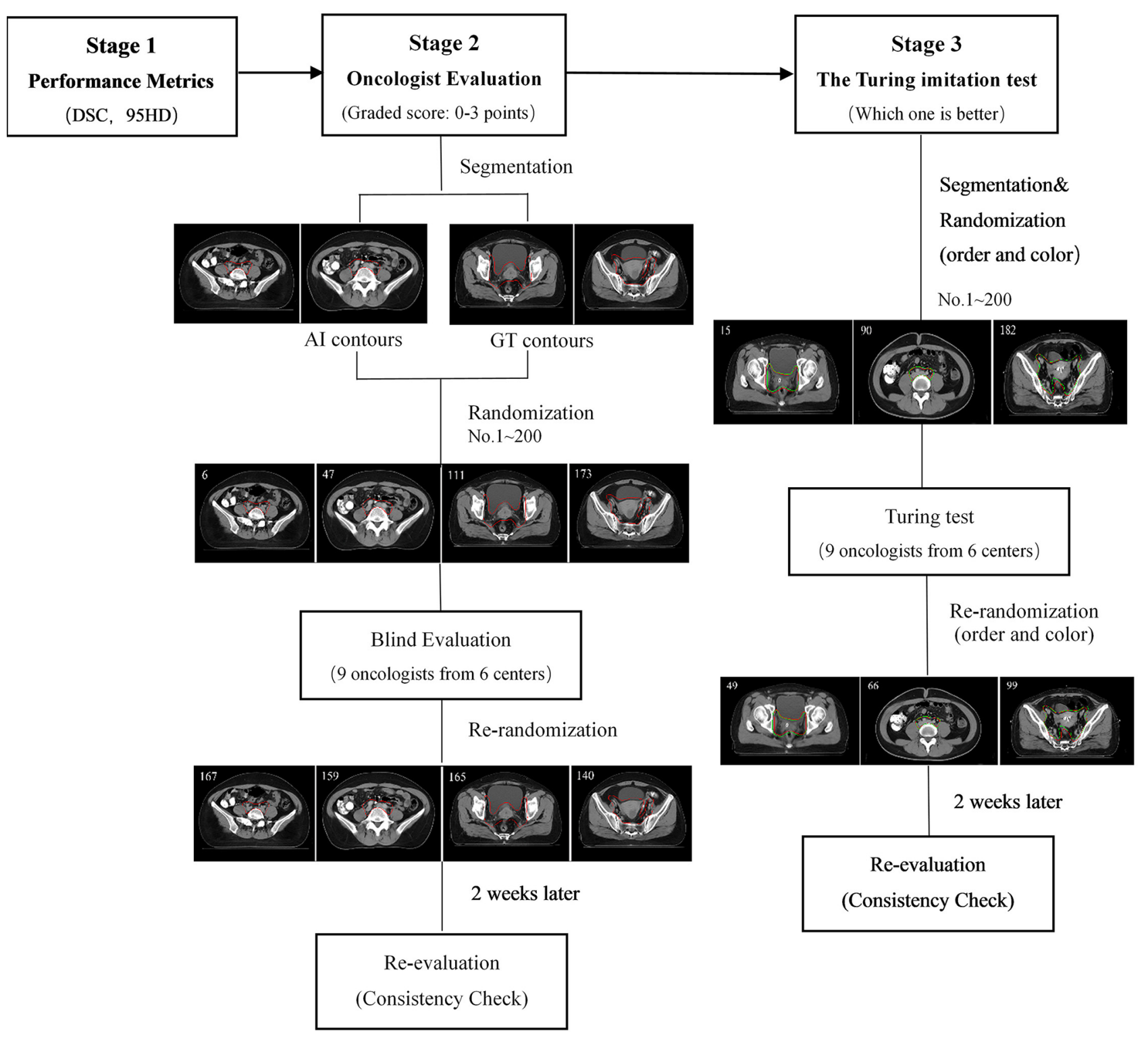

FIGURE 2 | The flowchart of the three-stage multicenter randomized controlled evaluation.

TABLE 1 | Criteria for the radiation oncologist evaluation.

\begin{tabular}{|c|c|c|}
\hline Score & Grade & Criteria \\
\hline 3 & No revision & The segmentation is perfect and completely acceptable for treatment. \\
\hline 2 & Minor revision & The segmentation needs a few minor edits but has no significant clinical impact without correction. \\
\hline 1 & Major revision & The segmentation needs significant revision. Treatment planning should not proceed without contour correction. \\
\hline 0 & Rejection & The segmentation is unacceptable and needs to be redrawn. \\
\hline
\end{tabular}

The steps are outlined as follows:

1. Data acquisition: Twenty slices containing CTV from 10 patients' planning CT scans were randomly selected to generate a CT dataset consisting of 200 axial slices.
2. Segmentation: Both machine AI and human GT contours were generated for each dataset. Ten slices of each patient were randomly selected and overlaid with AI contours, while the other 10 slices were overlaid with GT contours. The contour color of the two groups was intentionally made the same for the blind test. 
3. Randomization: The 200 CT slices were randomized by Fisher-Yates shuffle with an assigned unique ID so that the study authors could later distinguish whether each contour was an AI or GT.

4. Blind evaluation: The dataset of 200 randomized slices were distributed to the nine radiation oncologists. Each slice was scored from 0 to 3 blindly.

5. Consistency evaluation: After 2 weeks, the same dataset assigned in a new random order was distributed to the nine radiation oncologists for a second grading.

6. Analysis: The mean scores and the percentage of clinical acceptance of the AI and GT groups were calculated.

\section{Stage 3: The Turing Imitation Test}

The Turing imitation test is a subjective head-to-head comparison between GT- and AI-generated contours. In this test, the participant was presented with two contours overlaid simultaneously in the same CT slice, one of which was generated by the AI. The radiation oncologist was requested to choose which contour was better for clinical application. The steps are outlined as follows:

1. Data acquisition: We randomly extracted $20 \mathrm{CTV}$ containing axial CT slices from each of the remaining 10 test patients to generate a 200 -slice dataset.

2. Segmentation: For each slice, the AI and GT contours of CTV were generated randomly in a different color (red or green). The structure colors were randomized on a per-slice basis so as not to bias the Turing imitation test.

3. Randomization: AI- and GT-generated CTV slices were randomized by Fisher-Yates shuffle and anonymized to facilitate the blind evaluation. Each slice was assigned a unique ID so images could be de-anonymized later to analyze.

4. Turing test: The dataset was distributed to the test team, consisting of nine radiation oncologists from six different centers. Each radiation oncologist was requested to compare the AI and GT delineations and select the one that was more suitable for clinical application. The evaluation time for each slice was limited to $30 \mathrm{~s}$ to prevent the observer from seeking additional visual clues regarding the source of the contour.

5. Consistency evaluation: After 2 weeks, the same dataset assigned in a new random order and color was distributed to the radiation oncologists for a new comparison.

6. Analysis: If the AI contours received a better evaluation, the result would be considered positive. The positive rates of the entire test set and of each oncologist were calculated. Following the original Turing proposal (24), the threshold of the overall positive result rate was set to $30 \%$. Above that, the AI model is considered to have passed the Turing imitation test.

\section{Statistical Analysis}

The mean and standard deviation of DSC and 95HD were calculated. The Wilcoxon matched-pairs signed-rank test was used to compare the AI and GT contours in the oncologist evaluation and the Turing imitation test. The score difference between AI and GT contours evaluated by each oncologist was performed by Mann-Whitney $U$ test. The Wilcoxon paired signed-rank test was used to compare the agreement of the oncologist evaluation between 2 weeks for each oncologist. The McNemar test was used to compare the consistency of the Turing test between 2 weeks. Statistical significance was set at two-tailed $P<.05$.

\section{RESULTS}

\section{Stage 1: Quantitative Performance Metrics}

All slices of the 20 testing patients were evaluated with the quantitative performance metrics, which is shown and compared with DpnUNet in Table 2. The DSC and 95HD values of the proposed model were $0.88 \pm 0.03$ and $3.46 \pm 1.88$ $\mathrm{mm}$, respectively.

\section{Stage 2: Oncologist Evaluation}

Table 3 shows oncologist evaluation results of CTV contours. Score $\geq 2$ was defined as suitable for clinical application. Using these scoring criteria for contour evaluation, most CTV contours were clinically acceptable by all the oncologists. For AI contours, the percentage of clinically acceptable scores was $97.4 \%$, compared to the $98.3 \%$ of GT contours. We also compared AI and GT scores with a separate Mann-Whitney test for each oncologist and found that there was no significant difference between the week 0 timepoint and the after-2-weeks timepoint. Figure 3 shows the CTV scores for AI and GT contours. The overall average scores for AI and GT were $2.68 v$ s. 2.71 in week 0 $(P=.206)$ and $2.62 v s .2 .63$ in week $2(P=.552)$, respectively. The intra-observer consistency analyses between 2 weeks were performed by the Wilcoxon paired signed-rank test. It was found that the consistency of two oncologists was poor, while the others had good consistency between 2 weeks $(P>.05)$.

\section{Stage 3: The Turing Imitation Test}

When considering physician selection of the AI contour as preferred over the GT contour as a positive result, the overall positive rate in week 0 was $54.17 \%$ compared with $45.83 \%$ negative rate $(P=.139)$, while in week 2 the positive rate was $54 \% v s$. the negative rate of $46 \%(P=.128)$, which demonstrated the proposed deep machine learning model performed equally well or even better than human delineation. Furthermore, the consistency evaluation was performed by repeating the same dataset in different random order and colors to the test team after 2 weeks. The results are shown in Table 4 . Subclass analysis was performed to evaluate individual oncologists and CT slices. The results showed that six slices $(3.0 \%$ in week 0$)$ of AI contours were scored to be better than GT by all the oncologists. The percentage of AI contours that were approved to be better by $\geq 5$ oncologists was $60.0 \%$ in week 0 and $42.5 \%$ in week 2 . The distribution map is shown in Figure 4. Sample CTV delineations are presented in Figure 5. 
TABLE 2 | The comparison of DSC and 95HD value of our proposed model and DpnUNet.

\begin{tabular}{|c|c|c|c|c|c|}
\hline \multirow[t]{2}{*}{ Test } & \multirow[t]{2}{*}{ Patient (No.) } & \multicolumn{2}{|c|}{ Proposed Model } & \multicolumn{2}{|c|}{ DpnUNet } \\
\hline & & DSC & 95HD (mm) & DSC & 95HD (mm) \\
\hline Stage 2 patient cohort : & 1 & 0.9 & 1.95 & 0.84 & 2.09 \\
\hline \multirow[t]{9}{*}{ Oncologist Evaluation } & 2 & 0.91 & 2.34 & 0.84 & 2.38 \\
\hline & 3 & 0.9 & 3.68 & 0.89 & 3.61 \\
\hline & 4 & 0.9 & 1.95 & 0.90 & 1.85 \\
\hline & 5 & 0.83 & 7.68 & 0.75 & 8,84 \\
\hline & 6 & 0.88 & 2.98 & 0.81 & 3.10 \\
\hline & 7 & 0.84 & 7.07 & 0.80 & 8.10 \\
\hline & 8 & 0.9 & 2.55 & 0.93 & 2.45 \\
\hline & 9 & 0.89 & 2.83 & 0.83 & 3.85 \\
\hline & 10 & 0.88 & 3.35 & 0.86 & 3.41 \\
\hline Stage 3 patient cohort : & 11 & 0.85 & 5.1 & 0.75 & 6.17 \\
\hline \multirow{10}{*}{ The Turing Test } & 12 & 0.91 & 2.83 & 0.90 & 3.48 \\
\hline & 13 & 0.81 & 7.76 & 0.84 & 7.92 \\
\hline & 14 & 0.91 & 2.24 & 0.89 & 2.33 \\
\hline & 15 & 0.91 & 2.21 & 0.94 & 1.97 \\
\hline & 16 & 0.89 & 2.24 & 0.87 & 2.06 \\
\hline & 17 & 0.9 & 2.83 & 0.82 & 2.49 \\
\hline & 18 & 0.89 & 2.45 & 0.92 & 2.88 \\
\hline & 19 & 0.93 & 2.25 & 0.94 & 2.26 \\
\hline & 20 & 0.85 & 2.93 & 0.84 & 2.25 \\
\hline & Mean \pm STD & $0.88 \pm 0.03$ & $3.46 \pm 1.88$ & $0.86 \pm 0.06$ & $3.67 \pm 2.22$ \\
\hline
\end{tabular}

TABLE 3 | Graded oncologist evaluation for Al and GT contours.

Week 0

\begin{tabular}{|c|c|c|c|c|c|c|c|c|c|c|c|c|c|c|c|c|c|c|}
\hline \multirow{2}{*}{$\begin{array}{l}\text { Oncologist } \\
\text { Score }\end{array}$} & \multicolumn{2}{|c|}{ A } & \multicolumn{2}{|c|}{ B } & \multicolumn{2}{|c|}{ C } & \multicolumn{2}{|c|}{ D } & \multicolumn{2}{|c|}{ E } & \multicolumn{2}{|c|}{$\mathbf{F}$} & \multicolumn{2}{|c|}{ G } & \multicolumn{2}{|c|}{$\mathbf{H}$} & \multicolumn{2}{|c|}{ I } \\
\hline & Al & GT & Al & GT & Al & GT & Al & GT & Al & GT & Al & GT & Al & GT & Al & GT & Al & GT \\
\hline 3 & $89 \%$ & $97 \%$ & $93 \%$ & $95 \%$ & $30 \%$ & $37 \%$ & $71 \%$ & $74 \%$ & $54 \%$ & $57 \%$ & $75 \%$ & $85 \%$ & $94 \%$ & $94 \%$ & $82 \%$ & $77 \%$ & $45 \%$ & $37 \%$ \\
\hline 2 & $11 \%$ & $3 \%$ & $7 \%$ & $5 \%$ & $61 \%$ & $56 \%$ & $28 \%$ & $25 \%$ & $46 \%$ & $42 \%$ & $25 \%$ & $15 \%$ & $6 \%$ & $6 \%$ & $18 \%$ & $23 \%$ & $45 \%$ & $57 \%$ \\
\hline 1 & 0 & 0 & 0 & 0 & 9\% & $6 \%$ & $1 \%$ & $1 \%$ & 0 & $1 \%$ & 0 & 0 & 0 & 0 & 0 & 0 & $10 \%$ & $6 \%$ \\
\hline 0 & 0 & 0 & 0 & 0 & 0 & $1 \%$ & 0 & 0 & 0 & 0 & 0 & 0 & 0 & 0 & 0 & 0 & 0 & $0 \%$ \\
\hline Mean Score & 2.89 & 2.96 & 2.93 & 2.95 & 2.21 & 2.29 & 2.70 & 2.73 & 2.54 & 2.56 & 2.75 & 2.85 & 2.94 & 2.94 & 2.82 & 2.77 & 2.35 & 2.31 \\
\hline$P$ value & 0.061 & & 0.553 & & 0.282 & & 0.640 & & 0.719 & & 0.078 & & 1.000 & & 0.382 & & 0.494 & \\
\hline & & & & & & & & Week 2 & & & & & & & & & & \\
\hline 3 & $93 \%$ & $92 \%$ & $88 \%$ & $93 \%$ & $29 \%$ & $37 \%$ & $78 \%$ & $77 \%$ & $42 \%$ & $50 \%$ & $78 \%$ & $69 \%$ & $94 \%$ & $96 \%$ & $33 \%$ & $33 \%$ & $50 \%$ & $42 \&$ \\
\hline 2 & $7 \%$ & $8 \%$ & $12 \%$ & $7 \%$ & $63 \%$ & $54 \%$ & $22 \%$ & $21 \%$ & $57 \%$ & $50 \%$ & $22 \%$ & $31 \%$ & $5 \%$ & $4 \%$ & $62 \%$ & $64 \%$ & $39 \%$ & $56 \%$ \\
\hline 1 & 0 & 0 & 0 & 0 & $8 \%$ & $9 \%$ & 0 & $1 \%$ & $1 \%$ & 0 & 0 & 0 & $1 \%$ & 0 & $5 \%$ & $3 \%$ & $7 \%$ & $2 \%$ \\
\hline 0 & 0 & 0 & 0 & 0 & 0 & 0 & 0 & 0 & 0 & 0 & 0 & 0 & 0 & 0 & 0 & 0 & $4 \%$ & 0 \\
\hline Mean Score & 2.93 & 2.92 & 2.88 & 2.93 & 2.21 & 2.28 & 2.78 & 2.74 & 2.41 & 2.5 & 2.78 & 2.69 & 2.93 & 2.96 & 2.28 & 2.3 & 2.35 & 2.40 \\
\hline$P$ value & 0.789 & & 0.229 & & 0.352 & & 0.940 & & 0.230 & & 0.150 & & 0.509 & & 0.846 & & 0.728 & \\
\hline Consistency ( $P$ value) & & 0.782 & & 0.108 & & 0.907 & & 0.064 & & 0.007 & & 0.118 & & 0.491 & & 0.000 & & 0.170 \\
\hline
\end{tabular}

$P<0.05$, the results are statistically significant. 


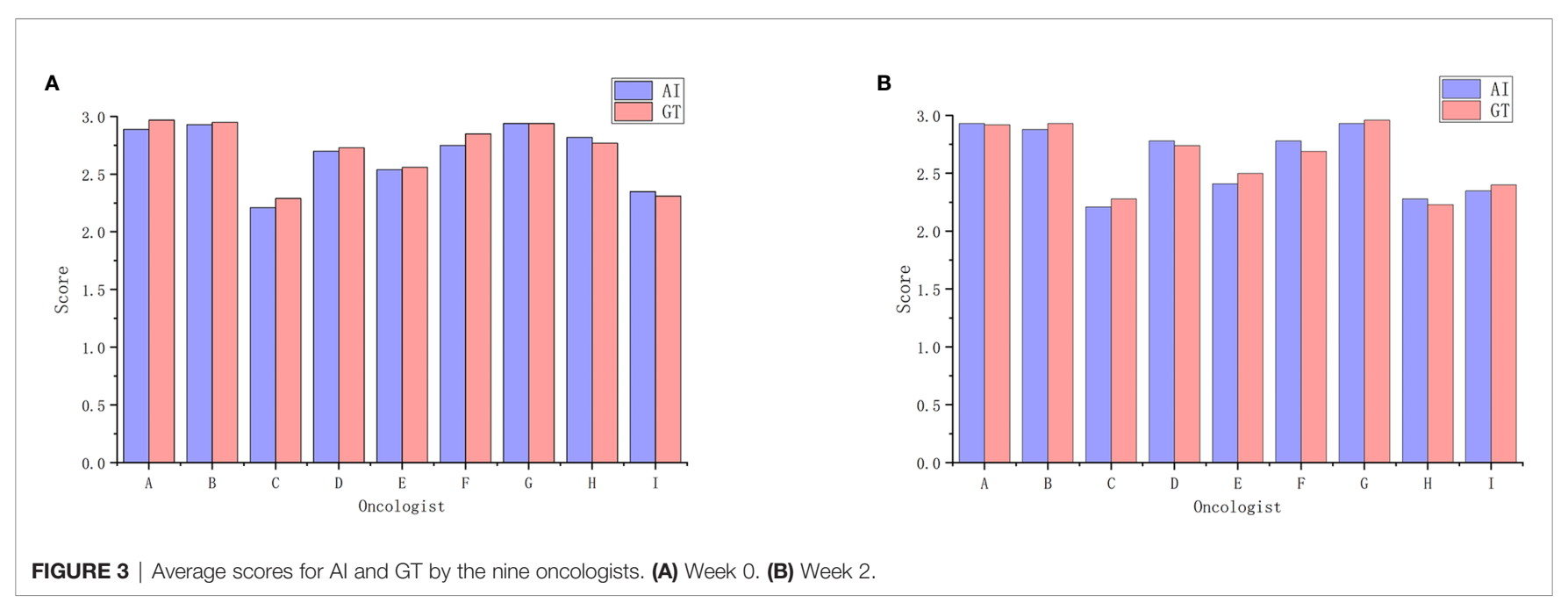

TABLE 4 | The results of the Turing-like imitation test.

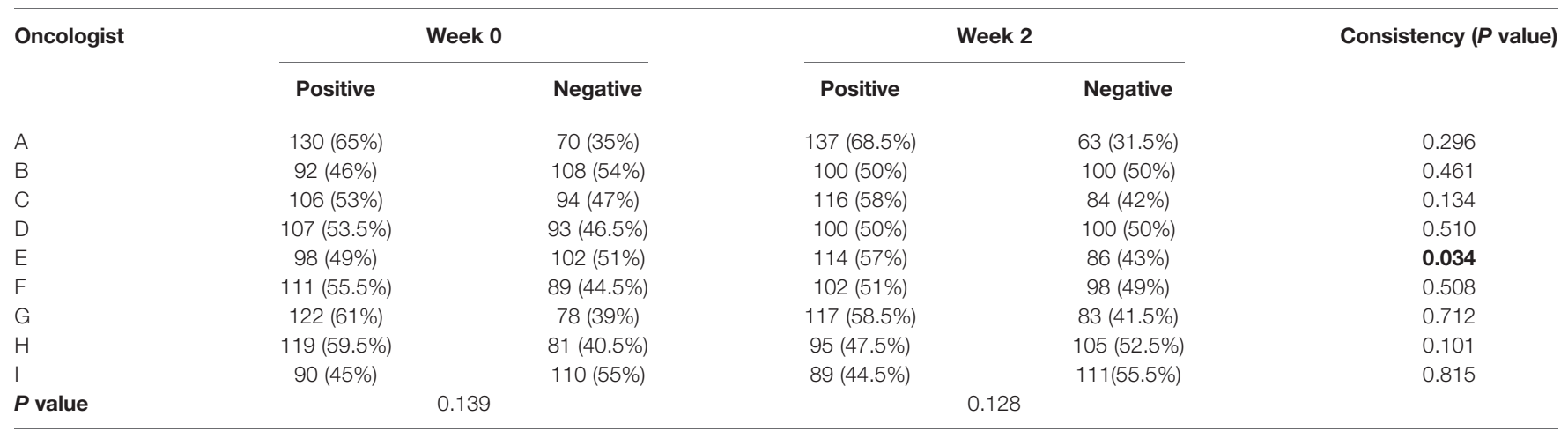

$P<0.05$, results are statistically significant.

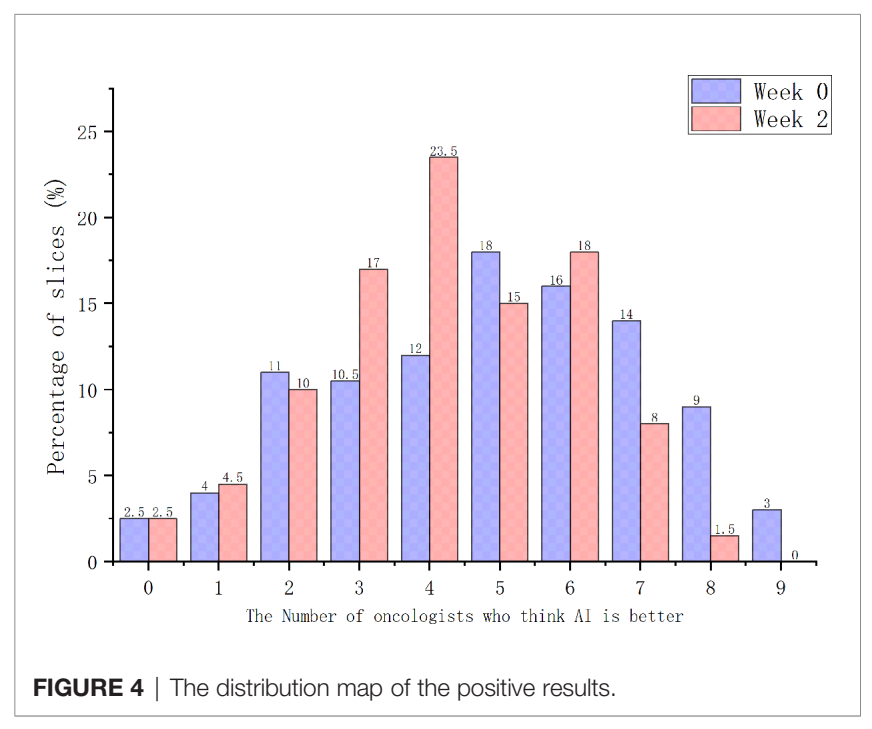

\section{DISCUSSION}

Segmentation of CTV is an essential step for successful radiotherapy delivery (16). However, manual delineation is time-consuming and subjective, with considerable inter- and intra-observer variability (25-28). Therefore, accurate and consistent automated segmentation methods are highly desirable and useful for pretreatment radiotherapy planning. Automatic segmentation techniques especially based on CNN models have made significant progress with increasing reliability and accuracy in recent years, thus potentially relieving radiation oncologists from the time-cost of contouring. To the authors' knowledge, very few studies were reported on the automatic delineation of the CTV (2932) due to the ambiguous and blurred boundaries between the CTV and normal tissues, the potential for tumor spread or subclinical diseases in the CT images, and the inter-observer variability in recognition of anatomical structures. The current most common approach to evaluate automatic delineation of the CTV is to compare with GT contours using quantitative measures such as DSC and HD $(33,34)$. However, this mathematical evaluation is basic and depends only on the geometrical properties of the organ being delineated. This approach does not incorporate clinical judgment and may not adequately extract the main characteristics and the core elements of the image.

Given the clinical application, an authors' previous study added subjective oncologist evaluation to the proposed model, 

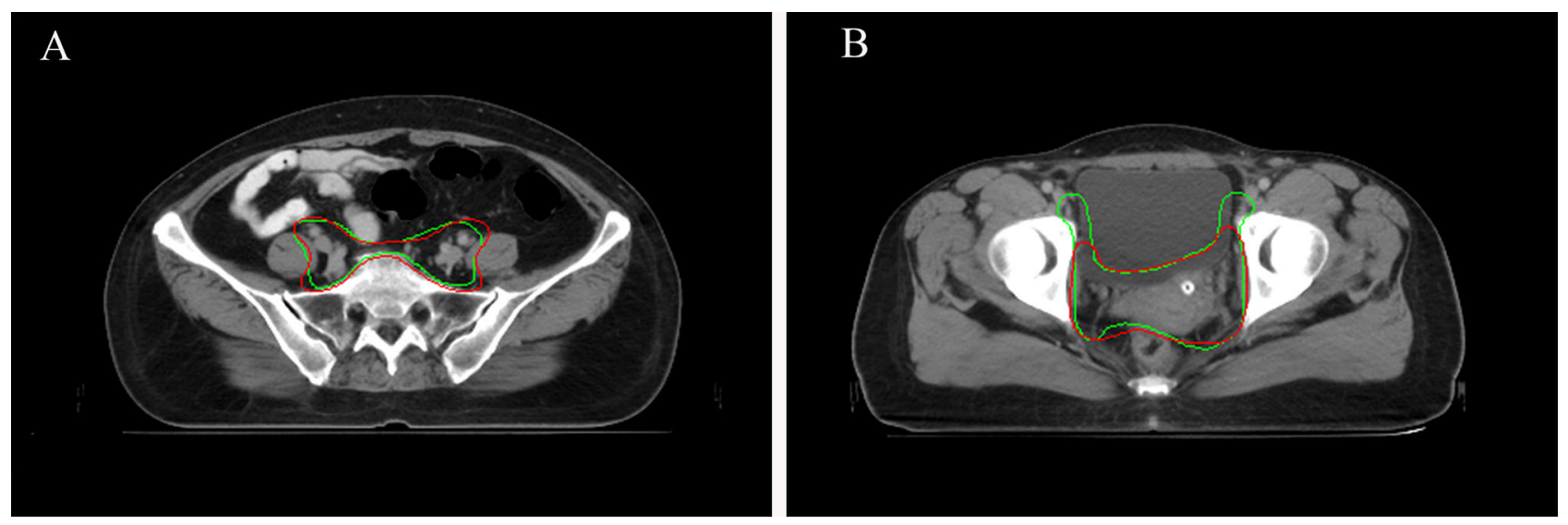

FIGURE 5 | (A) Sample CTV where the Al contour was approved by all the oncologists. Al contours in green line. GT contours in red line. (B) Sample CTV where the GT contour was approved by all the oncologists. Al contours in green line. GT contours in red line.

and the result showed that more than $88 \%$ of the slices predicted from DpnUNet were evaluated as "No revision" or "Minor revision" (13). However, when radiation oncologists were presented with AI and GT contours overlaid simultaneously in the same CT slice, the GT contour was always the one chosen to be better. Therefore, a novel auto-segmentation model that indeed performs comparably well or even better to manual delineation for CTV delineation is desirable. Moreover, the current evaluating performance of segmentation, particularly CTV segmentation, can be challenging due to the large variations among different centers and observers $(35,36)$. Therefore, a three-stage randomized controlled evaluation framework was proposed, combining the three elements of traditional performance metrics, oncologist evaluation, and the Turing imitation test, for a comprehensive assessment of the proposed model in cervical cancer CTV segmentation.

During stage- 1 evaluation, the mean DSC value of CTV of the proposed model was 0.88 , which was higher compared with the acceptable threshold of 0.80 to 0.86 used in other studies $(13,37$ 39). The average 95HD value was $3.46 \mathrm{~mm}$ compared to $5.34 \mathrm{~mm}$ by the DpnUNet model (13). The results indicated a strong concordance between the proposed automatic model and human experts for CTV contouring.

In stage-2 evaluation, a multicenter randomized controlled evaluation involving nine radiation oncologists from six different centers was designed to examine the model's clinical utility and generalization. The anonymized CT slices were randomly distributed with AI or GT contours to experienced radiation oncologists for assessment. The choice of a random design instead of using entire connected slices is mainly because AI sometimes has obvious characteristics at certain levels, especially at the beginning and the end, which do not affect the accuracy of target delineation but make it more easy to be distinguished. Moreover, the evaluation is more clinically relevant and minimizes assessment bias as oncologists are blinded to the source of the contours. The results showed that our proposed model was highly acceptable for clinical application and treatment. There was no significant difference in physician acceptability ratings between scores of AI and GT contours, which means our model can provide consistent segmentation and performed well with good agreement to the manual contours. However, there were still $2.6 \%$ of cases where the AI contours were judged by some oncologists to require major revision. We retrospectively analyzed these outlier cases and found that most of them were in the middle level of the pelvic cavity; thus, the ROIs had very unclear boundaries and massive diversity of sizes, shapes with low contrast to the rectum, bladder, and small intestines. The circumstances mentioned above limit the generalizability of the AI model, and therefore more caution is warranted.

In 1950, Alan Turing proposed an influential test for how to evaluate artificial intelligence: an imitation is successful when we cannot distinguish it from the real thing (24). Here, this analogous logic was applied to the artificial segmentation technology, and a similar Turing imitation test was proposed. The variant of the Turing imitation test used in this study is a randomized blinded evaluation. In contrast with the stage- 2 task, in which evaluators viewed individual stimuli and made categorical judgments, the radiation oncologists were presented with AI and GT contour masks on the same slice and were requested to choose which was better. If the positive rate of $\mathrm{AI}$ is more than $30 \%$, then the AI model was considered to have passed the test. It is a straightforward head-to-head comparison, which compares two contours in the exact same condition to minimize the interference factors such as scanning conditions, anatomical variations, and severity of disease in different patients. As shown in Table 4, the segmentation model passed the Turing test with overall positive rates much higher than $30 \%$. The overall positive rate was $54.17 \%$ in week 0 and $54 \%$ in week 2, which demonstrated that the AI segmentation model performed equally well as humans $(P=.139, P=.128)$. Moreover, correlations were observed between the objective and subjective 
measures. Those with lower DSC and 95HD values were also more likely to be flagged as requiring revision or inferior performance during the subjective evaluation.

Subjective assessment still has drawbacks. Oncologists involved in this study stated that they might change their opinion of the grading score if they viewed it at a later point, and may not be able to definitively decide between two contours if they showed a high degree of overlap. Therefore, the intraobserver consistency analyses between 2 weeks were performed during stage- 2 and stage-3 evaluation. Most oncologists were found to maintain good consistency between 2 weeks without significant difference. Considering that good scores or positive rates could have resulted from a range of factors affecting how the contours were evaluated, a distribution map across all images involved in the Turing imitation test was additionally generated, to evaluate the number of oncologists who consistently thought AI contours were better. The results showed that the percentage of AI contours to be better than GT by $\geq 5$ oncologists was $60.0 \%$ in week 0 and $42.5 \%$ in week 2 , which further demonstrated the excellent performance of the proposed segmentation model.

\section{CONCLUSION}

In this study, a novel deep-learning-based CNN model for fully automatic and accurate CTV segmentation in cervical cancer was proposed. Then a comprehensive three-stage randomized controlled evaluation framework was performed to validate the model. This evaluation system is a combination of objective and subjective evaluation and can diminish the risk of bias and enhance real-world clinical relevance compared to the most commonly used evaluation method of applying performance metrics alone. The tested AI model was demonstrated to be accurate and comparable to the manual CTV segmentation in cervical cancer patients. Furthermore, this study provided guidelines for each step, which can be referred to by other centers according to their sample size limitation. While this study focuses only on cervical cancer, the methodology and general learnings may translate to other tumor sites. Moreover, this comprehensive assessment of contouring performance may also be referenced as a base framework for evaluating the clinical utility of automatic segmentation methods in the future.

\section{REFERENCES}

1. Koh WJ, Abu-Rustum NR, Bean S, Bradley K, Campos SM, Cho KR, et al. Cervical Cancer, Version 3.2019, NCCN Clinical Practice Guidelines in Oncology. J Natl Compr Cancer Network (2019) 17:64-84. doi: 10.6004/ jnccn.2019.0001

2. Chibwesha CJ, Stringer JSA. Cervical Cancer as a Global Concern: Contributions of the Dual Epidemics of HPV and HIV. Jama (2019) 322:1558-60. doi: 10.1001/jama.2019.16176

3. Peters WA, Liu PY, Barrett RJ, Stock RJ, Monk BJ, Berek JS, et al. Concurrent Chemotherapy and Pelvic Radiation Therapy Compared With Pelvic Radiation Therapy Alone as Adjuvant Therapy After Radical Surgery in High-Risk Early-Stage Cancer of the Cervix. J Clin Oncol (2000) 18:1606-13. doi: $10.1200 /$ JCO.2000.18.8.1606

\section{DATA AVAILABILITY STATEMENT}

The original contributions presented in the study are included in the article/Supplementary Material. Further inquiries can be directed to the corresponding authors.

\section{ETHICS STATEMENT}

The evaluation was reviewed and approved by the Peking Union Medical College Hospital Institutional Review board. The patients/participants provided their written informed consent to participate in this study. Written informed consent was obtained from the individual(s) for the publication of any potentially identifiable images or data included in this article.

\section{AUTHOR CONTRIBUTIONS}

FZ and JQ had full access to all of the data in the study and take responsibility for the integrity of the data and the accuracy of the data analysis. Concept and design: FZ, JQ, ZKL, WC. Acquisition, analysis, or interpretation of data: HG, HZ, JS, XL, JG, JY, WW, ZYL, YZ, YYC, JD. AI model design: SW, QC, YC. Drafting of the manuscript: WC, ZKL. Critical revision of the manuscript for important intellectual content: AL, RL. All authors contributed to the article and approved the submitted version.

\section{FUNDING}

This work was funded by the following grants from the Non-profit Central Research Institute Fund of Chinese Academy of Medical Sciences (grant number 2019XK320014). FZ takes responsibility for the integrity of the data and the accuracy of the data analysis.

\section{SUPPLEMENTARY MATERIAL}

The Supplementary Material for this article can be found online at: https://www.frontiersin.org/articles/10.3389/fonc.2021.702270/ full\#supplementary-material

4. Han K, Milosevic M, Fyles A, Pintilie M, Viswanathan AN. Trends in the Utilization of Brachytherapy in Cervical Cancer in the United States. Int J Radiat Oncol Biol Phys (2013) 87:111-9. doi: 10.1016/j.ijrobp.2013.05.033

5. Monk BJ, Tewari KS, Koh WJ. Multimodality Therapy for Locally Advanced Cervical Carcinoma: State of the Art and Future Directions. J Clin Oncol (2007) 25:2952-65. doi: 10.1200/JCO.2007.10.8324

6. Sarıül M, Ozyildirim BM, Avci M. Differential Convolutional Neural Network. Neural Networks (2019) 116:279-87. doi: 10.1016/j.neunet. 2019.04.025

7. Esteva A, Robicquet A, Ramsundar B, Kuleshov V, DePristo M, Chou K, et al. A Guide to Deep Learning in Healthcare. Nat Med (2019) 25:24-9. doi: 10.1038/s41591-018-0316-Z

8. Lin L, Dou Q, Jin YM, Zhou GQ, Tang YQ, Chen WL, et al. Deep Learning for Automated Contouring of Primary Tumor Volumes by MRI for 
Nasopharyngeal Carcinoma. Radiology (2019) 291:677-86. doi: 10.1148/ radiol.2019182012

9. Lustberg T, van Soest J, Gooding M, Peressutti D, Aljabar P, van der Stoep J, et al. Clinical Evaluation of Atlas and Deep Learning Based Automatic Contouring for Lung Cancer. Radiother Oncol (2018) 126:312-7. doi: 10.1016/j.radonc.2017.11.012

10. Ahn SH, Yeo AU, Kim KH, Kim C, Goh Y, Cho S, et al. Comparative Clinical Evaluation of Atlas and Deep-Learning-Based Auto-Segmentation of Organ Structures in Liver Cancer. Radiat Oncol (2019) 14:213. doi: 10.1186/s13014019-1392-z

11. Ayyalusamy A, Vellaiyan S, Subramanian S, Ilamurugu A, Satpathy S, Nauman M, et al. Auto-Segmentation of Head and Neck Organs at Risk in Radiotherapy and Its Dependence on Anatomic Similarity. Radiat Oncol J (2019) 37:134-42. doi: 10.3857/roj.2019.00038

12. Kim N, Chang JS, Kim YB, Kim JS. Atlas-Based Auto-Segmentation for Postoperative Radiotherapy Planning in Endometrial and Cervical Cancers. Radiat Oncol (2020) 15:106. doi: 10.1186/s13014-020-01562-y

13. Liu Z, Liu X, Guan H, Zhen H, Sun Y, Chen Q, et al. Development and Validation of a Deep Learning Algorithm for Auto-Delineation of Clinical Target Volume and Organs at Risk in Cervical Cancer Radiotherapy. Radiother Oncol (2020) 153:172-9. doi: 10.1016/j.radonc.2020.09.060

14. van Rooij W, Dahele M, Ribeiro Brandao H, Delaney AR, Slotman BJ, Verbakel WF. Deep Learning-Based Delineation of Head and Neck Organs at Risk: Geometric and Dosimetric Evaluation. Int J Radiat Oncol Biol Phys (2019) 104:677-84. doi: 10.1016/j.ijrobp.2019.02.040

15. van der Veen J, Willems S, Deschuymer S, Robben D, Crijns W, Maes F, et al. Benefits of Deep Learning for Delineation of Organs at Risk in Head and Neck Cancer. Radiother Oncol (2019) 138:68-74. doi: 10.1016/j.radonc.2019.05.010

16. Men K, Zhang T, Chen X, Chen B, Tang Y, Wang S, et al. Fully Automatic and Robust Segmentation of the Clinical Target Volume for Radiotherapy of Breast Cancer Using Big Data and Deep Learning. Phys Med (2018) 50:13-9. doi: 10.1016/j.ejmp.2018.05.006

17. Men K, Chen X, Zhang Y, Zhang T, Dai J, Yi J, et al. Deep Deconvolutional Neural Network for Target Segmentation of Nasopharyngeal Cancer in Planning Computed Tomography Images. Front Oncol (2017) 7:315. doi: 10.3389/fonc. 2017.00315

18. Moeskops P, Veta M, Lafarge MW, Eppenhof KAJ, Pluim JPW. Adversarial Training and Dilated Convolutions for Brain MRI Segmentation In: Deep Learning in Medical Image Analysis and Multimodal Learning for Clinical Decision Support. Springer: Cham. (2017) p. 56-64. doi: 10.1007/978-3-3196755897

19. Norman B, Pedoia V, Majumdar S. Use of 2D U-Net Convolutional Neural Networks for Automated Cartilage and Meniscus Segmentation of Knee MR Imaging Data to Determine Relaxometry and Morphometry. Radiology (2018) 288:177-85. doi: 10.1148/radiol.2018172322

20. Zhou LQ, Wu XL, Huang SY, Wu GG, Ye HR, Wei Q, et al. Lymph Node Metastasis Prediction From Primary Breast Cancer US Images Using Deep Learning. Radiology (2020) 294:19-28. doi: 10.1148/radiol.2019190372

21. Harris VA, Staffurth J, Naismith O, Esmail A, Gulliford S, Khoo V, et al. Consensus Guidelines and Contouring Atlas for Pelvic Node Delineation in Prostate and Pelvic Node Intensity Modulated Radiation Therapy. Int J Radiat Oncol Biol Phys (2015) 92:874-83. doi: 10.1016/j.ijrobp.2015.03.021

22. Lim K, Small W Jr, Portelance L, Creutzberg C, Jürgenliemk-Schulz IM, Mundt A, et al. Consensus Guidelines for Delineation of Clinical Target Volume for Intensity-Modulated Pelvic Radiotherapy for the Definitive Treatment of Cervix Cancer. Int J Radiat Oncol Biol Phys (2011) 79:348-55. doi: 10.1016/j.ijrobp.2009.10.075

23. Small WJr., Bosch WR, Harkenrider MM, Strauss JB, Abu-Rustum N, Albuquerque KV, et al. NRG Oncology/RTOG Consensus Guidelines for Delineation of Clinical Target Volume for Intensity Modulated Pelvic Radiation Therapy in Postoperative Treatment of Endometrial and Cervical Cancer: an Update. Int J Radiat Oncol Biol Phys (2021) 109:413-24. doi: 10.1016/j.ijrobp.2020.08.061

24. Turing AM. Computing Machinery and Intelligence. Mind (1950) 433-60. doi: 10.1093/mind/LIX.236.433

25. Keenan LG, Rock K, Azmi A, Salib O, Gillham C, McArdle O. An Atlas to Aid Delineation of Para-Aortic Lymph Node Region in Cervical Cancer: Design and Validation of Contouring Guidelines. Radiother Oncol (2018) 127:417-22. doi: 10.1016/j.radonc.2018.02.013

26. Small WJr., Mell LK, Anderson P, Creutzberg C, De Los Santos J, Gaffney D, et al. Consensus Guidelines for Delineation of Clinical Target Volume for Intensity-Modulated Pelvic Radiotherapy in Postoperative Treatment of Endometrial and Cervical Cancer. Int J Radiat Oncol Biol Phys (2008) 71:428-34. doi: 10.1016/j.ijrobp.2007.09.042

27. Apolle R, Appold S, Bijl HP, Blanchard P, Bussink J, Faivre-Finn C, et al. Inter-Observer Variability in Target Delineation Increases During Adaptive Treatment of Head-and-Neck and Lung Cancer. Acta Oncol (2019) 58:137885. doi: 10.1080/0284186X.2019.1629017

28. Bø HK, Solheim O, Jakola AS, Kvistad KA, Reinertsen I, Berntsen EM. IntraRater Variability in Low-Grade Glioma Segmentation. J Neuro-oncol (2017) 131:393-402. doi: 10.1007/s11060-016-2312-9

29. Larsson R, Xiong JF, Song Y, Ling-Fu, Chen YZ, Xiaowei X, et al. Automatic Delineation of the Clinical Target Volume in Rectal Cancer for Radiation Therapy Using Three-Dimensional Fully Convolutional Neural Networks. Annu Int Conf IEEE Eng Med Biol Soc (2018) 2018:5898-901. doi: 10.1109/ EMBC.2018.8513506

30. Song $\mathrm{Y}, \mathrm{Hu}$ J, Wu Q, Xu F, Nie S, Zhao Y, et al. Automatic Delineation of the Clinical Target Volume and Organs at Risk by Deep Learning for Rectal Cancer Postoperative Radiotherapy. Radiother Oncol (2020) 145:186-92. doi: 10.1016/j.radonc.2020.01.020

31. Shusharina N, Söderberg J, Edmunds D, Löfman F, Shih H, Bortfeld T. Automated Delineation of the Clinical Target Volume Using Anatomically Constrained 3D Expansion of the Gross Tumor Volume. Radiother Oncol (2020) 146:37-43. doi: 10.1016/j.radonc.2020.01.028

32. Liu Z, Liu X, Xiao B, Wang S, Miao Z, Sun Y, et al. Segmentation of Organs-atRisk in Cervical Cancer CT Images With a Convolutional Neural Network. Phys Med (2020) 69:184-91. doi: 10.1016/j.ejmp.2019.12.008

33. Vrtovec T, Močnik D, Strojan P, Pernuš F, Ibragimov B. Auto-Segmentation of Organs at Risk for Head and Neck Radiotherapy Planning: From AtlasBased to Deep Learning Methods. Med Phys (2020) 47(9):e929-50. doi: $10.1002 / \mathrm{mp} .14320$

34. van Dijk LV, Van den Bosch L, Aljabar P, Peressutti D, Both S, Steenbakkers RJHM, et al. Improving Automatic Delineation for Head and Neck Organs at Risk by Deep Learning Contouring. Radiother Oncol (2020) 142:115-23. doi: 10.1016/j.radonc.2019.09.022

35. Louie AV, Rodrigues G, Olsthoorn J, Palma D, Yu E, Yaremko B, et al. InterObserver and Intra-Observer Reliability for Lung Cancer Target Volume Delineation in the 4D-CT Era. Radiother Oncol (2010) 95:166-71. doi: 10.1016/j.radonc.2009.12.028

36. Federico M, Hernandez-Socorro CR, Ribeiro I, Martin JG, Oramas MDR, Saez-Bravo ML, et al. Prospective Intra/Inter-Observer Evaluation of PreBrachytherapy Cervical Cancer Tumor Width Measured in TRUS and MR Imaging. Radiat Oncol (2019) 14:173. doi: 10.1186/s13014-019-1352-7

37. Kurata Y, Nishio M, Kido A, Fujimoto K, Yakami M, Isoda H, et al. Automatic Segmentation of the Uterus on MRI Using a Convolutional Neural Network. Comput Biol Med (2019) 114:103438. doi: 10.1016/j.compbiomed.2019.103438

38. Chen L, Shen C, Zhou Z, Maquilan G, Albuquerque K, Folkert MR, et al. Automatic PET Cervical Tumor Segmentation by Combining Deep Learning and Anatomic Prior. Phys Med Biol (2019) 64:085019. doi: 10.1088/13616560/ab0b64

39. Ghose S, Holloway L, Lim K, Chan P, Veera J, Vinod SK, et al. A Review of Segmentation and Deformable Registration Methods Applied to Adaptive Cervical Cancer Radiation Therapy Treatment Planning. Artif Intell Med (2015) 64:75-87. doi: 10.1016/j.artmed.2015.04.006

Conflict of Interest: Authors SW, QC, and YC were employed by the company MedMind Technology Co.

The remaining authors declare that the research was conducted in the absence of any commercial or financial relationships that could be construed as a potential conflict of interest.

Publisher's Note: All claims expressed in this article are solely those of the authors and do not necessarily represent those of their affiliated organizations, or those of the publisher, the editors and the reviewers. Any product that may be evaluated in 
this article, or claim that may be made by its manufacturer, is not guaranteed or endorsed by the publisher.

Copyright $\odot 2021$ Liu, Chen, Guan, Zhen, Shen, Liu, Liu, Li, Geng, You, Wang, Li, Zhang, Chen, Du, Chen, Chen, Wang, Zhang and Qiu. This is an open-access article distributed under the terms of the Creative Commons Attribution License (CC BY). The use, distribution or reproduction in other forums is permitted, provided the original author(s) and the copyright owner(s) are credited and that the original publication in this journal is cited, in accordance with accepted academic practice. No use, distribution or reproduction is permitted which does not comply with these terms. 